\title{
End-to-End Lung Cancer Diagnosis on Computed Tomography Scans using 3D CNN and Explainable AI
}

\author{
Chaitanya Rahalkar \\ Savitribai Phule Pune University
}

\author{
Anushka Virgaonkar \\ Savitribai Phule Pune University \\ Sumedh Patkar \\ Savitribai Phule Pune University
}

\author{
Dhaval Gujar \\ Savitribai Phule Pune University
}

\begin{abstract}
Lung cancer is one of the most common malignant neoplasms all over the world. It accounts for more cancer deaths than any other cancer. It is increasingly being recognized in hospitals all across the globe. With the increasing prevalence of smoking, Lung cancer has reached epidemic proportions. Thus, we propose a 3D-CNN-based model [6] that uses a patient's Computed Tomography scans to detect nodules and check for malignancy. We intend to add an explainable aspect to the result since the central problem of such models is that they are regarded as black-box models, and they lack an explicit declarative knowledge representation [9]. This calls for systems enabling to make decisions transparent, understandable and explainable.
\end{abstract}

\section{General Terms}

Deep Learning, Neural Networks, Machine Learning, Consumer Health

\section{Keywords}

Supervised Learning, Convolutional Neural Networks

\section{INTRODUCTION}

In India, millions of people are diagnosed with Lung diseases. In the diagnosis of the Lung disease, assessing the pattern, locating the pattern, and studying the regional distribution of involvement, is the domain of radiology|4]. However, there is a dearth of trained Radiologists that can accurately diagnose the likelihood of the disease. Furthermore, many of the current attempts at automating the process have not been intuitive enough for actual use by Radiologists in clinics. The application which we have proposed would allow Radiologists to perform seamless diagnosis and assessment.

This tool takes images of CT scans of a patient, which are stored in Digital Imaging and Communications in Medicine (DICOM) format [11], as input. It detects the nodules in the images. These nodules are a group of abnormal tissues that develop in the Lung. The nodules are classified as malignant or benign using a Deep Learning model. 3D Convolutional Neural Networks (CNNs) [7]are used for classifying the images as they have proven to show supremacy over the traditional neural network models. The output is a standardized report which presents a detailed diagnosis of the malig- nant nodules. This system is specifically designed to be used by Radiologists, who use medical imaging to diagnose diseases like Lung cancer. This tool provides a way for Radiologists to interact with the simple and easy to use web-based interface, allowing them to inspect the Lung nodules. The application generates output reports represented in Portable Document Format (PDF)[10][1].

\section{MOTIVATION}

With an estimated 160,000 deaths in 2018, Lung cancer is the most common cause of cancer death in the United States. Lung cancer screening using low-dose Computed Tomography has been shown to reduce mortality by $2043 \%$ and is now included in US screening guidelines. The CT scans show the presence of Lung nodules better than regular chest X-Rays. The CT scans represent a twodimensional view of a section or slice of the organ being examined. The three-dimensional shape can be constructed by arranging the CT scans according to their slice index. Applying 3D CNNs to the constructed three-dimensional medical image of the Lung results in the diagnosis of Lung nodules. Moreover, characteristics such as sphericity, spiculation, and texture of the detected nodules are also predicted to supplement the results.

\section{PREVIOUS WORKS}

There were different scenarios where detection of Lung cancer was done on Computed Tomography scans.

(1) One use case involved studying an individual patient's previous and current CT scans to predict the risk of Lung cancer using deep learning techniques. This model achieved state-ofthe-art performance. It shows a high potential for the use of deep learning models in predicting Lung cancer.

(2) Another use case for using Deep Learning to examine CT scans was initiated by the United States Preventive Services Task Force, which recommended continuous analysis of CT scans of the people with smoking history. This involved people who currently smoke or have quit within the past 15 years. The initiative was taken to educate the population about the need for regular screening to promote informed decision making.

(3) Lung-RADS is a classification system that is used for managing Lung cancer screening results. The classification system generates various categories corresponding to the probability 
of Lung cancer. A study was conducted to quantify the accuracy of assigning the Lung-RADS categories to screening CT scans. A high interobserver agreement was observed.

\section{RESEARCH ASSUMPTIONS}

The following assumptions were made based on our observations in collecting the data and training the model for classification:

(1) All the CT scan images are in the standardized DICOM [3] [2] format. It is a structured file format to store raw medical scans along with their metadata. Any scan not conforming to this format is likely to be predicted incorrectly by the algorithm.

(2) The resolution of the CT scans conforms to the scans taken from the LUNA 16 dataset [3]. The accuracy is considerably affected if the scan does not comply with the specified dimension details.

\section{APPLICATION SCOPE}

The scope of the decision-support system is to aid Radiologists in testing CT scans to diagnose Lung cancer. The system aims to expedite the screening process while providing a seamless experience for any Radiologist. A facility to systematically store detailed patient records and fetch them as required.

\section{DETECTION FRAMEWORK}

The fully automated Lung cancer diagnosis system consists mainly of two parts: nodule detection and classification. We designed a 3D Faster R-CNN for nodule detection, as shown in Fig 1

\begin{tabular}{|c|c|c|}
\hline Stage & Output & Weights \\
\hline $\begin{array}{l}\text { Pre-dual } \\
\text { path }\end{array}$ & $96 \times 96 \times 96,24$ & $3 \times 3 \times 3,24$ \\
\hline $\begin{array}{l}\text { Dual path } \\
\text { block } 1\end{array}$ & $48 \times 48 \times 48,48$ & $\left\{\begin{array}{l}1 \times 1 \times 1,24 \\
3 \times 3 \times 3,24,(\text { stride } 2) \\
1 \times 1 \times 1,32\end{array}\right\} \times 2$ \\
\hline $\begin{array}{l}\text { Dual path } \\
\text { block } 2\end{array}$ & $24 \times 24 \times 24,72$ & $\left\{\begin{array}{l}1 \times 1 \times 1,48 \\
3 \times 3 \times 3,48,(\text { stride } 2) \\
1 \times 1 \times 1,56\end{array}\right\} \times 2$ \\
\hline $\begin{array}{l}\text { Dual path } \\
\text { block } 3\end{array}$ & $12 \times 12 \times 12,96$ & $\left\{\begin{array}{l}1 \times 1 \times 1,72 \\
3 \times 3 \times 3,72,(\text { stride } 2) \\
1 \times 1 \times 1,80\end{array}\right\} \times 2$ \\
\hline $\begin{array}{l}\text { Dual path } \\
\text { block } 4\end{array}$ & $6 \times 6 \times 6,120$ & $\left\{\begin{array}{l}1 \times 1 \times 1,96 \\
3 \times 3 \times 3,96,(\text { stride } 2) \\
1 \times 1 \times 1,104\end{array}\right\} \times 2$ \\
\hline Deconv. 1 & $12 \times 12 \times 12,216$ & $2 \times 2 \times 2,216$ \\
\hline $\begin{array}{l}\text { Dual path } \\
\text { block } 5\end{array}$ & $12 \times 12 \times 12,152$ & $\left\{\begin{array}{l}1 \times 1 \times 1,128 \\
3 \times 3 \times 3,128 \\
1 \times 1 \times 1,136\end{array}\right.$ \\
\hline Deconv. 2 & $24 \times 24 \times 24,224$ & $2 \times 2 \times 2,152$ \\
\hline $\begin{array}{l}\text { Dual path } \\
\text { block } 6\end{array}$ & $24 \times 24 \times 24,248$ & $\left\{\begin{array}{l}1 \times 1 \times 1,224 \\
3 \times 3 \times 3,224 \\
1 \times 1 \times 1,232\end{array}\right.$ \\
\hline Output & $24 \times 24 \times 24,3 \times 5$ & $\begin{array}{l}\text { Dropout, } \mathrm{p}=0.5 \\
1 \times 1 \times 1,64 \\
1 \times 1 \times 1,15\end{array}$ \\
\hline
\end{tabular}

Fig. 1. Neural Network Architecture

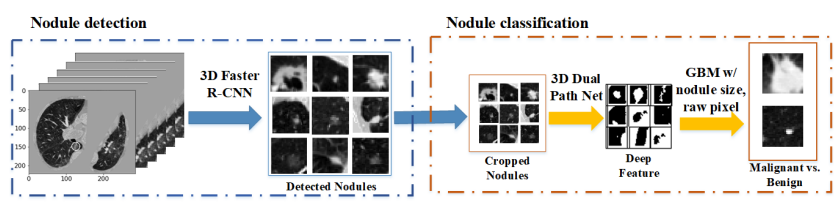

Fig. 2. End-to-end Pipeline

As shown in Fig. 2 the nodule detection module and the classification module are pipelined, making the entire diagnosis system completely streamlined.

\subsection{Dataset}

The Lung Nodule Analysis 2016 (LUNA 2016) dataset consists of 888 annotated CT scans. The dataset is used to train the convolutional neural network, which can then identify cancerous cells from normal cells, which is the main task of our decision-support system. A web-based system is added on top of this prediction API to detect nodules, classify them as malignant or benign, and apply Explainable-AI [8], to explain the reasons for the classified outcomes in a human-understandable manner. Existing challenges in the dataset include inter-grader variability and high false-positive and false-negative rates.

\section{DESIGN OF THE SYSTEM}

\subsection{System Architecture}

The overall application is broken down into several broad sections. The following is a high-level view of the component hierarchy:

(1) The job of the prediction service (via the trained models it wraps) is to implement these core predictive tasks and expose them for use. The models themselves shouldn't need to know about the prediction service, which in turn should not need to know anything about the interface of the application. These services are loosely coupled with each other providing considerable granularity and modularity.

(2) The job of the interface backend (API) is to ferry data back and forth between the client browser and model service, handle web requests, take care of computationally intensive transformations not appropriate for frontend Javascript, and persist user-entered data to a data store. It should not need to know much about the interface frontend, but its main job is to relay data for frontend manipulation, so it's acceptable for this part to less abstractly generalizable than the prediction service.

(3) The job of the interface frontend (UI) is to demonstrate as much value as possible by exposing functionality that the models make possible in an intuitive and attractive format.

Each of these jobs are represented in the architecture diagram shown in Fig. 3

\subsection{User Interfaces}

Imagery - Interface:

(1) List available DICOM images on the local filesystem.

(2) Return a preview of the DICOM image on the local filesystem as an HTML-displayable image.

(3) Allow a specific image to be selected and create a new "Case" to work on for the remainder of the steps. 


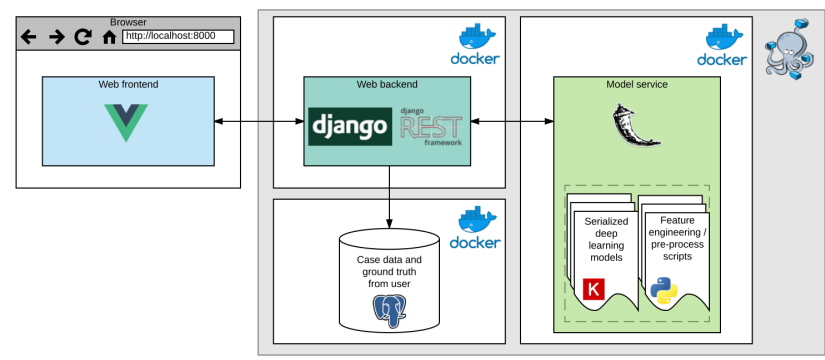

Fig. 3. System Architecture

(4) This image will be used in the identification step next.

Detect - Interface:

(1) Act as the data broker for the image being worked on (selected in our current session), requesting predictions from the Prediction service along with filtering or other passed parameters and relay them back to the frontend.

(2) Provide an endpoint that can receive a payload of all nodule locations, (1) nodule centroid location tuple (X \# voxels from left, Y \# voxels from the top, $\mathrm{Z}$ as slice number), (2) whether the nodule was predicted or manually added, and (3) whether the nodule was marked for further analysis or not (those manually identified by the user are presumably concerning)[5].

(3) List all available predicted candidate sites. Allow each to be selected to view details.

(4) When a candidate is selected, show a detail view in an image viewer control. By default, show the slice containing the predicted centroid and a marker indicating where the predicted centroid occurs.

(5) Allow the user to navigate through the imagery freestyle and mark other locations that the models missed.

(6) When the user has finished marking candidates, send all of this labelled data to the backend in the format specified above.

Annotate - Interface:

(1) List nodules identified as part of the current case for detailed view.

(2) Receive and persist to the database a Radiologist-supplied label for how concerning each nodule is.

(3) Receive and persist to the database the other fields expected for each case in the RSNA Radiology Reporting Template for CT Lung Cancer Screening.

Segment - Prediction:

(1) Given a nodule centroid location tuple (X No. of voxels from left, Y No. of voxels from the top, $\mathrm{Z}$ as slice number), return a 3D boolean mask with true values for voxels associated with that nodule and false values for other tissue or voids.

(2) For any given slice of any given nodule, transform the true values in the binary mask from the Prediction service into a series of vertices defining an irregular polygon that can be displayed on the image. In the transformation to vertices, take a precision parameter which causes the transformation to use more or fewer vertices to make the polygon fit the binary mask more precisely.
(3) Given a stack of such irregular polygons, calculate several summary statistics of interest, to include: a new estimated centroid location tuple (X No. of voxels from left, Y No. of voxels from the top, $\mathrm{Z}$ as slice number); a volume in cubic millimetres; a longest axial diameter.

(4) Provide an endpoint that allows the current vertices to be persisted to the database attached to the current case and nodule.

Results - Interface:

(1) Summarize all of the data from the case, including the general notes and details for each nodule, into a single JSON report.

(2) Allow export of the whole report in different formats.

\subsection{Functional Requirements}

(1) Identification: The "Region of Interest" to be identified is the nodule, whose presence is an indication of Lung cancer. The system can successfully differentiate the nodule from the tissue in the background and pinpoints its location in the image.

(2) Classification: The classification model will classify the nodule's type: Malignant or Benign. It performs analysis on the past labelled data to predict the label of the present data. The risk factor associated with the prediction is calculated.

(3) Segmentation: The system must find the boundaries of nodules in the image. Accurate representation of the same will be displayed on the frontend web application. The system helps Radiologists refine and build out the computer-aided diagnosis with the help of automatic measures.

(4) Data Preprocessing: The data preprocessing module will apply filters to the input DICOM images. Resizing algorithms are applied for further processing. The slicing factor of frames is selected and fixed.

(5) Query Response: The response to the client's queries will be shown by a direct action on a rendered DICOM image having bounding boxes that indicate the presence of nodules. Metadata is displayed for each image.

\subsection{Deployment Architecture}

The deployment architecture used is a client-server architecture. The server is either hosted on a cloud-based platform or is hosted locally. To serve the website, the Django web framework is used. The inference and training modules require the use of a GPU. The classification and training modules make use of Tensorflow. PostgreSQL database is stored to store the data. The client-side of the architecture is the client web application, instances of which are divided into authorized units for query processing and one for admin usage. This deployment model is shown in Fig. 4

\subsection{Data Flow Model}

Detection of nodules on a selected DICOM image is carried out using the neural network architecture, and the risk factor is estimated for various parameters concerning malignancy. The features of the nodules are then displayed along with their positional values. Radiologists can add their risk estimates and observations. A report is generated that follows a standardized template. The data can be exported for record-keeping and follow up. A diagrammatic representation of this model is seen in Fig. 5 


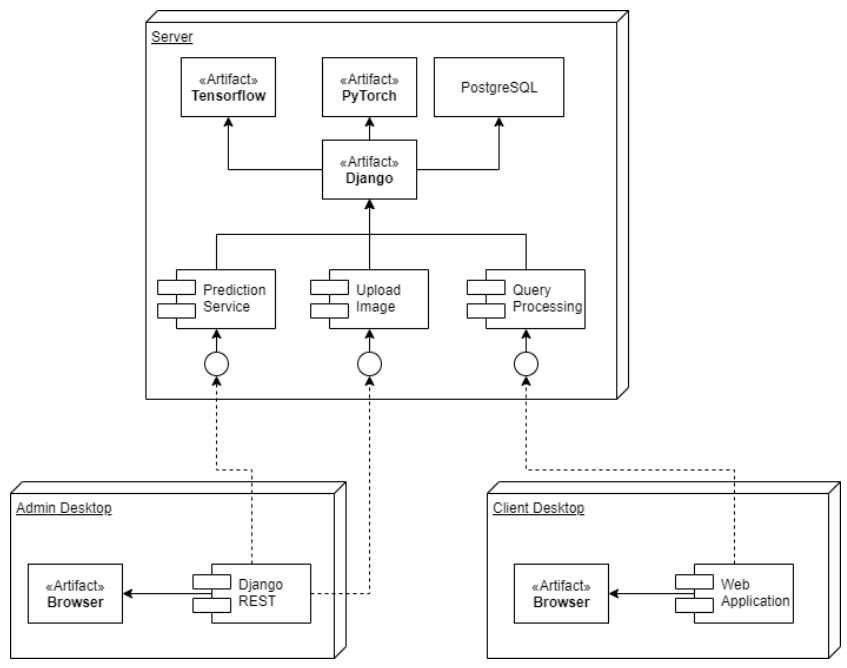

Fig. 4. Deployment Diagram

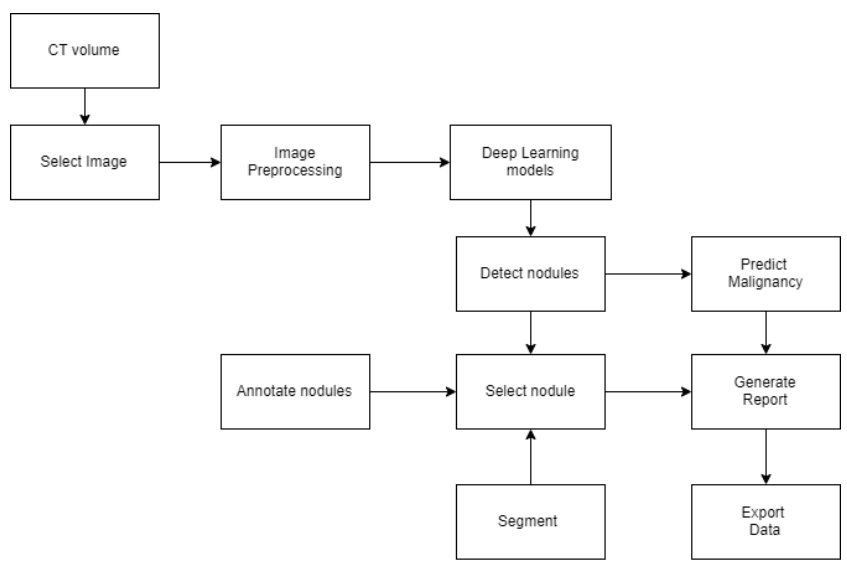

Fig. 5. Data Flow

\section{IMPLEMENTATION DETAILS}

The project design is broadly divided into the following modules, each responsible for handling different tasks :

\subsection{Nodule Detection Module}

The nodules detected in the previous module are displayed to the Radiologist in this module. They are arranged in descending order of their risk factor. The Radiologist gets an option to dismiss a nodule if he/she feels that the nodule has been incorrectly identified or mark it as concerning. The UI screen for the Nodule Detection Module is shown in Fig. 6

\subsection{Annotation and Segmentation Module}

The Annotation and Segmentation Module deals with each individual nodule in more detail. It gives the Radiologist detailed options to further describe the nodule's nature, add notes to the report, and most importantly, it allows the Radiologist to label the nodule's

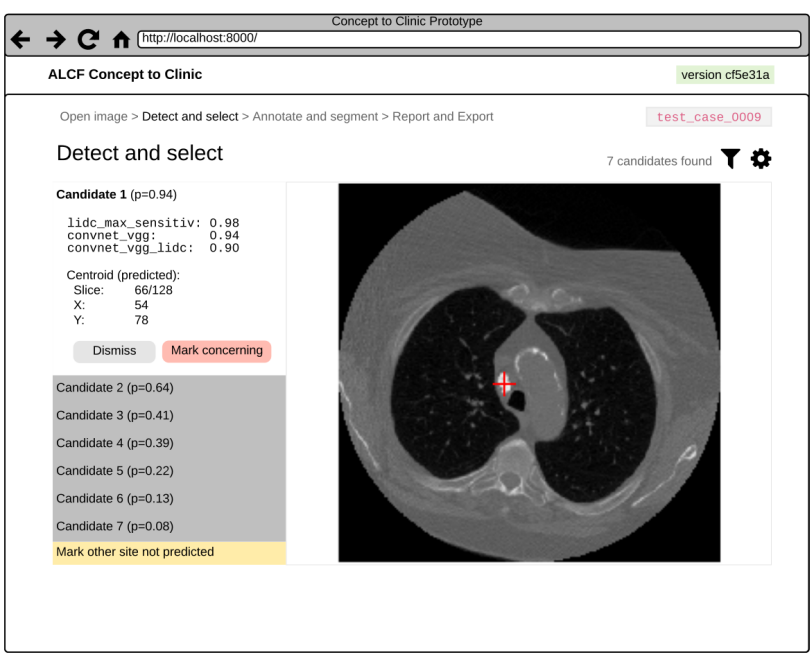

Fig. 6. Nodule Detection

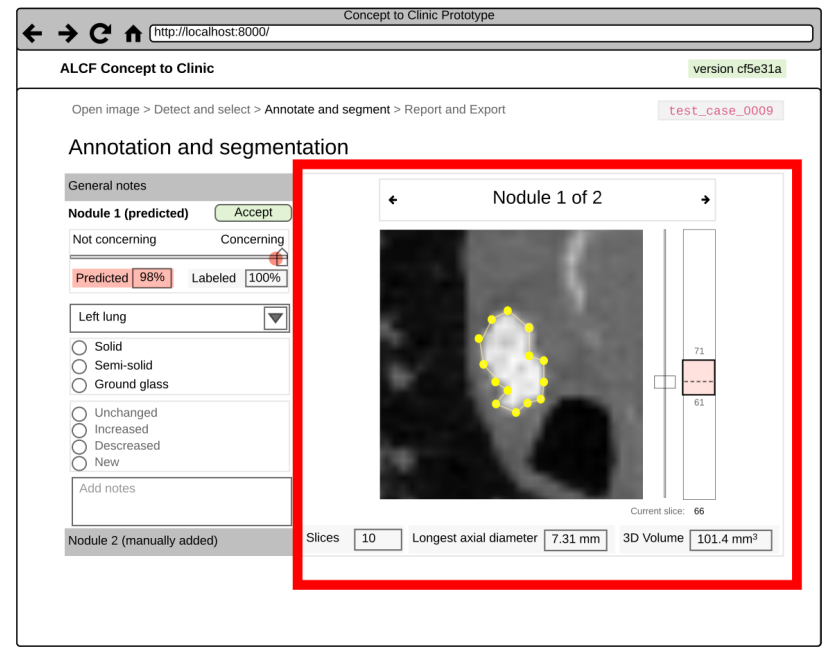

Fig. 7. Annotate Nodule

concerning scale. This allows the model to be improved. The corresponding screen for this module is shown in Fig.7

\subsection{Report Generation Module}

An important last step in medical care is report generation, for doctors and Radiologists around the world to be able to view and understand the report. For this, standardized reports by the RSNA (Radiological Society of North America) and ACR (American College of Radiology) have been implemented. This is shown in Fig. 8.

\subsection{Patient Case Management Module}

As shown in the representational view in Fig. 9 this support module is designed to be used by the Radiologist to easily sort and find previously taken CT scans from a single, convenient user interface and not having to jump between applications and re-import or re- 


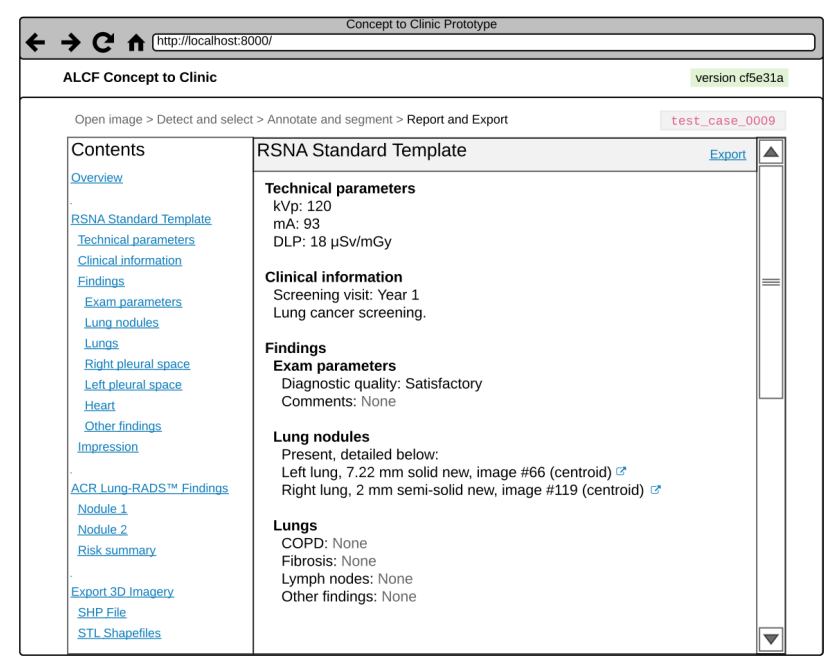

Fig. 8. Generating Standardized Reports

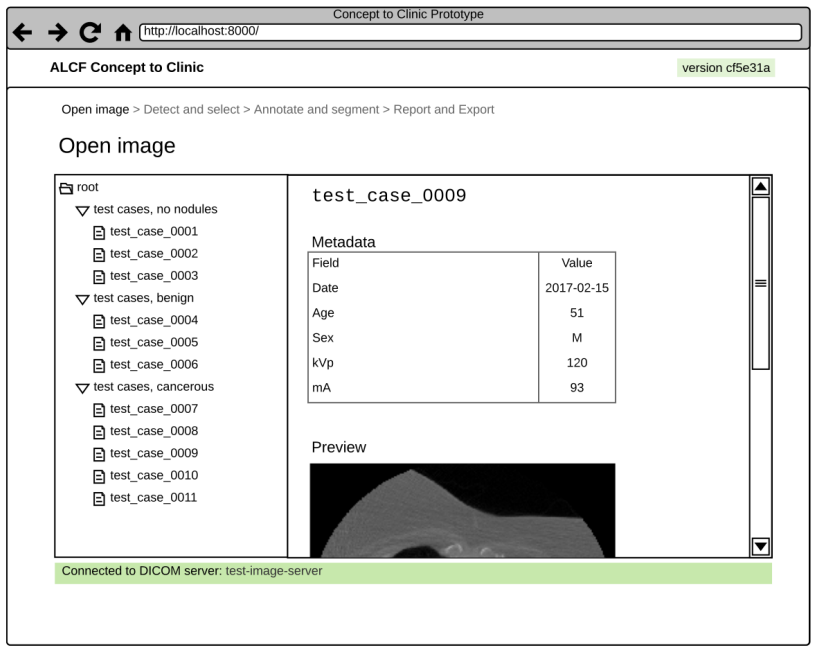

Fig. 9. Managing Patient Cases

Table 1.

Accuracy

\begin{tabular}{c|c|c|c|c|c}
\hline & Dr. 1 & Dr. 2 & Dr. 3 & Dr. 4 & Average \\
\hline Doctors & 92.44 & 93.61 & 93.82 & 88.13 & 91.44 \\
\hline Proposed Model & 94.05 & 94.01 & 92.16 & 91.19 & $\mathbf{9 2 . 8 8}$ \\
\hline
\end{tabular}

export. Metadata information and a quick preview of the CT scan is made available in the right pane for easier finding of records.

\section{RESULTS}

We compared our predictions with those of four "simulated" experienced doctors on their individually confident nodules(with an individual score). Comparison results are concluded in Table 1 . Fig 10 shows the bounding box generated for the DICOM image, indicating the detected nodule.
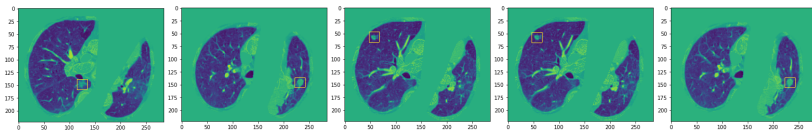

Fig. 10. Nodule Detection

\section{FUTURE SCOPE}

The current implementation is restricted only to a naive segmented $3 \mathrm{D}-\mathrm{CNN}$-based approach. The prediction and annotation accuracy and the area under the ROC curve can be considerably improved by using other novel algorithmic implementations. Furthermore, hyperparameter tuning can allow the system to extend its capabilities beyond the current state-of-the-art implementation[10].

\section{CONCLUSION}

The system comprises of a unified interface for Radiologists and cancer researchers that automates the CT scan based Lung cancer detection process. A complete end-to-end system is constructed that has the following steps in the architectural pipeline:

Creating a patient case by uploading the CT scan of the patient. Automated detection of Lung nodules in the sliced CT scan raw model, using 3D CNNs. Providing support to manually add nodules undetected by the algorithm.

Annotating detected Lung nodules and providing scope for Radiologists to manually change the concerning percentage predicted by the algorithm.

Generate standardized reports complying with the RSNA standard template.

\section{ACKNOWLEDGEMENTS}

We would like to take this opportunity to thank our guide, Dr R. B. Ingle, for giving us all the help and guidance we needed. We are grateful to him for his kind support. His valuable suggestions were very helpful.

We are also grateful to Prof. M. S. Takalikar, Head of Computer Engineering Department, PICT for her indispensable support and suggestions.

In the end, our special thanks to the college for providing various resources such as laboratory with all needed software platforms and continuous Internet connection for our project.

\section{REFERENCES}

[1] D. R. Aberle et al. Reduced lung-cancer mortality with lowdose computed tomographic screening. pages 395-409. 365, 2011.

[2] The Data Science Bowl. 2017.

[3] The Luna16 Challenge. 2016.

[4] T. Chen and C. XGBoost: A Guestrin. Scalable tree boosting system. In Proc. 22nd SIGKDD Conference on Knowledge Discovery and Data Mining, 2016.

[5] E. R. DeLong, D. M. DeLong, and D. L. Clarke-Pearson. Comparing the areas under two or more correlated receiver operating characteristic curves: a nonparametric approach. Biometrics, pages 837-845, 1988. 
[6] Shuiwang Ji, Wei Xu, Ming Yang, and Kai Yu. 3d convolutional neural networks for human action recognition. IEEE Transactions on Pattern Analysis and Machine Intelligence, 35(1):221231, Jan 2013.

[7] J. Long, E. Shelhamer, and T. Darrell. Fully convolutional networks for semantic segmentation. CoRR, abs/, 1411:4038, 2014.

[8] S. J. Mason and N. E. Graham. Areas beneath the relative operating characteristics (roc) and relative operating levels (rol) curves: Statistical significance and interpretation. Quarterly Journal of the Royal Meteorological Society, 128:2145-2166, 2002.

[9] Marco Tulio Ribeiro, Sameer Singh, and Carlos Guestrin. "why should i trust you?": Explaining the predictions of any classifier. arXiv:1602.04938 [cs, stat], Aug 2016. arXiv: 1602.04938 .

[10] R. Siegel, K. Miller, and Jemal A. Cancer statistics. Ca cancer journal of clinicians 68. pages 7-30, 2018.

[11] The DICOM Standard. 